\title{
The ATLAS Semi-Conductor Tracker Operation and Performance
}

\author{
D.Robinson, on behalf of the ATLAS Collaboration \\ Cavendish Laboratory, University of Cambridge, Madingley Road, Cambridge, CB3 OHE, UK
}

\begin{abstract}
The Semi-Conductor Tracker (SCT) is a silicon strip detector and one of the key precision tracking devices in the Inner Detector of the ATLAS experiment at the CERN Large Hadron Collider (LHC). The SCT was installed and commissioned within ATLAS in 2007, and has been has been used to exploit fully the physics potential of the LHC since the first proton-proton collisions at $7 \mathrm{TeV}$ were delivered in 2009. In this paper, its operational status throughout data taking up to the end of 2011 is presented, and its tracking performance is reviewed.
\end{abstract}

Keywords: SCT, ATLAS, Silicon, Microstrip, Tracking, Radiation Damage

\section{Introduction}

The ATLAS Inner Detector (ID) is responsible for particle tracking in ATLAS and employs three different detector technologies: a pixel silicon detector closest to the interaction point, the SCT micro-strip silicon detector, and a Transition Radiation Tracker. The overall ID is $5.6 \mathrm{~m}$ long and $2.1 \mathrm{~m}$ in diameter, and resides in a $2 \mathrm{~T}$ axial magnetic field.

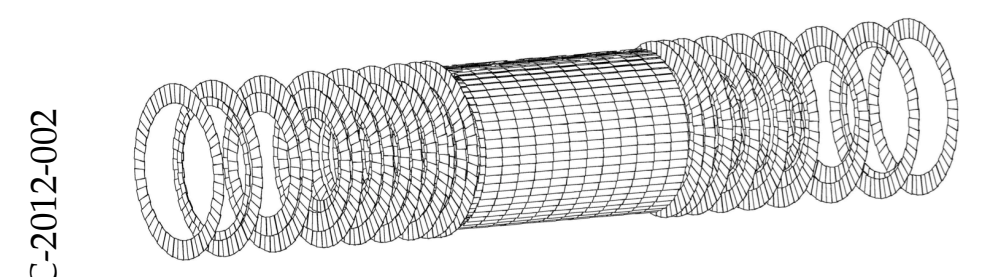

Figure 1: Geometrical layout of the SCT

Figure 1 illustrates the geometrical layout of the SCT, which was designed to ensure energetic particles pass through at least 4 silicon layers in the acceptance region. Closest to the interaction point are four concentric cylinders covering the pseudorapidity range $|\eta|<1.1-1.4$ and radii $30-51 \mathrm{~cm}$. On each side of the barrels is an end-cap comprising nine disks covering pseudorapidity range $1.1-1.4<|\eta|<2.5$ and radii extending to $56 \mathrm{~cm}$. The SCT comprises $61 \mathrm{~m}^{2}$ of silicon sensors with 6.3 million readout channels. It operates in a near zero humidity nitrogen environment and is cooled nominally to $-7^{\circ} \mathrm{C}$ by a $\mathrm{C}_{3} \mathrm{~F}_{8}$ evaporative cooling system, to suppress reverse annealing arising from radiation damage.

\subsection{The SCT sensors}

The silicon sensors[1] are all $285 \mu \mathrm{m}$ thick single-sided pon-n devices, with 768 AC-coupled micro-strips. There are 8448 identical rectangular shaped barrel sensors with size $64.0 \times 63.6 \mathrm{~mm}$ and $80 \mu \mathrm{m}$ strip pitch. The end cap disks use wedge shaped sensors of five slightly different sizes to accommodate the more complex geometry. There are 6944 wedge sensors in total, with strip pitch ranging from 56.9 to $90.4 \mu \mathrm{m}$.

\subsection{The Silicon Modules}

The basic granularity shown in Figure 1 is the silicon module. There are 2112 identical rectangular shaped modules[2] used to construct the barrel cylinders, and 1976 wedge shaped modules[3] of three different sizes used to construct the end-cap disks. Figure 2 shows photos of the end-cap and barrel modules. Modules consist of two pairs of back-to-back sensors, glued to a thermally conductive substrate for mechanical and thermal stability. Pairs on opposite sides are rotated by $40 \mathrm{mrad}$ to form a stereo angle in order to enable a resolution measurement in the direction parallel to the strips. The sensor micro-strips are wire bonded across to form effectively $12 \mathrm{~cm}$ long strips.
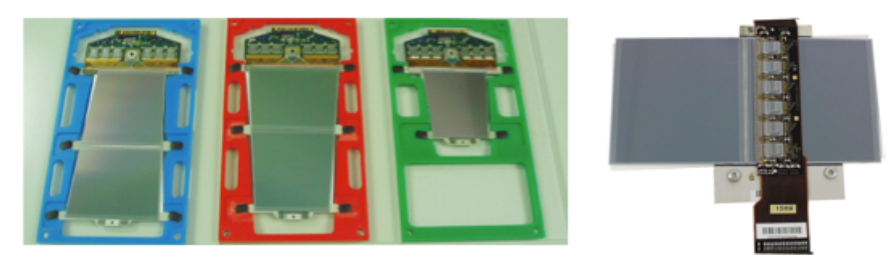

Figure 2: The SCT end-cap modules (left three images) and the barrel modules (right)

\subsection{ABCD Readout Chips}

Readout is via 128-channel ABCD[4] ASICs which are glued to a $\mathrm{Cu}$-polyimide flex circuit hybrid which forms a bridge across the top of the sensors. The ASICs were manufactured using the radiation-hard DMILL technology, and use a binary readout architecture with a $20 \mathrm{~ns}$ front end shaping time. A 
schematic illustrating the ABCD functionality is shown in Figure 3. The signal is shaped and amplified and applied to a comparator with programmable threshold, nominally set to $1 \mathrm{fC}$. The output of the comparator is strobed by the $40 \mathrm{MHz}$ LHC clock, and the binary output stored in a 132-deep pipeline which effectively stores the history of the comparator output for every clock cycle. Upon receipt of a trigger from the ATLAS trigger system, the contents of 3 pipeline bins are sampled. The SCT timing is optimised on a module-by-module basis to ensure hits from tracks from collisions result in a $01 \mathrm{X}$ occupancy pattern in the 3 sampled bins (no hit in first bin, hit in second bin and optional hit in third). The ABCD registers a 'hit' depending on the 3 bin occupancy pattern, which can be configured to ensure optimal efficiency depending on running conditions (eg 01X and $\mathrm{X} 1 \mathrm{X}$ for 25ns and 50ns LHC bunch trains respectively, or any hit pattern containing at least one ' 1 ' for cosmic rays).

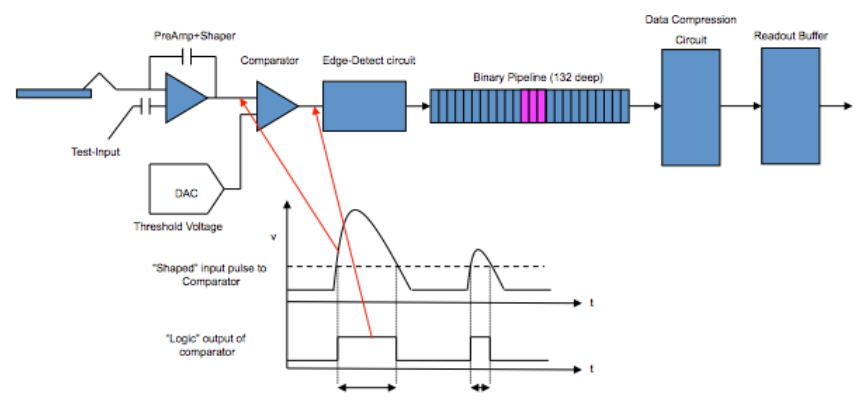

Figure 3: ABCD functionalilty schematic.

\subsection{Optical Links and Redundancy}

Communication to and from the modules is by optical links [5], referred to as TX links for transmission of trigger and command signals to the modules, and RX links for the transmission of data back from the modules. There is a single TX link per module, but two RX links for data transmission back from each side of the module. Off-detector and on-detector VCSEL arrays are used to broadcast data transmission for the TX and RX links respectively.

Redundancy options exist for both the TX and RX links, in case of fibre breaks, VCSEL deaths or IPIN diode problems. Redundancy is built into the TX system by having electrical links from one module to its neighbour. If a module loses its TX signal for any reason, an electrical control line can be set which will result in the neighbouring module sending a copy of its TX data to the module with the failed signal, without any impact on operation. A redundancy loop connects 12 adjacent modules in the barrels. For the end caps, the TX redundancy loops join modules around one ring, and connect 40 or 52 modules. For the data links, one side of the module can be configured to read out through the other link. Although use of RX redundancy inevitably reduces the readout bandwidth (due to the transmission of data from 12 chips instead of 6), this is still within limits for SCT operation. For the barrel modules, use of RX redundancy also results in the loss of one chip on the re-routed link.
Table 1: Typical use of redundancy of SCT optical links.

\begin{tabular}{|c|c|c|c|c|}
\hline Redundancy & Endcaps & Barrel & SCT & Fraction (\%) \\
\hline TX & 8 & 20 & 28 & 0.68 \\
RX link 0 & 35 & 13 & 48 & 1.17 \\
RX link 1 & 40 & 16 & 56 & 1.37 \\
\hline
\end{tabular}

A snapshot of use of TX and RX redundancy in the SCT is shown in Table 1 . The use of RX redundancy is relatively stable and numbers in the table are predominantly due to link failures during construction and integration of the SCT. The use of TX redundancy has varied widely during operations, mainly due to TX VCSEL deaths as described elsewhere in this paper.

\section{Operations}

LHC delivered $47 \mathrm{pb}^{-1}$ and $5.6 \mathrm{fb}^{-1}$ of proton-proton collision data at $7 \mathrm{TeV}$ in 2010 and 2011 respectively, as well as two 1 month periods of heavy ion collisions. The SCT has been fully operational throughout all data taking periods. It delivered high quality tracking data for $99.9 \%$ and $99.6 \%$ of the delivered luminosity in 2010 and 2011 respectively.

\subsection{Acceptance}

More than $99 \%$ of the SCT strips have remained fully functional and available for tracking at all times. Table 2 lists the

Table 2: Numbers of Disabled SCT Detector Elements.

\begin{tabular}{|c|c|c|c|c|}
\hline Component & Endcaps & Barrel & SCT & Fraction (\%) \\
\hline Modules & 20 & 10 & 30 & 0.73 \\
Strips & 6992 & 3681 & 10673 & 0.07 \\
Chips & 40 & 16 & 56 & 0.17 \\
\hline
\end{tabular}

typical numbers of disabled detector elements; the number of disabled strips (typically due to high noise or un-bonded channels) and non-functioning chips is negligible and the largest contribution is due to disabled modules, as detailed in Table 3. Half of the disabled modules are due to one cooling loop perma-

Table 3: Details of disabled modules

\begin{tabular}{|c|c|c|c|c|}
\hline & Endcaps & Barrel & SCT & Fraction (\%) \\
\hline Total & 20 & 10 & 30 & 0.73 \\
Fraction(\%) & 1.0 & 0.5 & 0.7 & \\
\hline Cooling & 13 & 0 & 13 & 0.3 \\
LV & 1 & 6 & 7 & 0.2 \\
HV & 5 & 1 & 6 & 0.2 \\
Readout & 1 & 3 & 4 & 0.1 \\
\hline
\end{tabular}

nently disabled as a result of an inaccessible leak in that loop. Fortunately this affects one quadrant of one of the outermost 
end-cap disks, and has negligible impact on tracking performance. The remaining modules are predominantly due to ondetector connection issues.

\subsection{Data taking efficiency}

The SCT Data Acquisition System[6] (DAQ) has proved to be highly reliable with excellent data taking efficiency. There are two potential sources of inefficiency: errors from the ABCD ASICs which flag that data fragments from those chips cannot be used for tracking, and a BUSY signal from the SCT DAQ that prevents ATLAS from taking data.

Chips may flag an error if they become desynchronised from the ATLAS system, or if their configuration becomes corrupt as a result of Single Event Upsets (SEUs). The rate of such errors is very low, but was further minimised in 2010 by the introduction of online monitoring of chip errors in the data and the automatic reconfiguration of the modules with those errors. In 2011, SCT implemented in addition an automatic global reconfiguration of all SCT module chips every $\sim 30$ minutes as a precaution against subtle deterioration in chip configurations as a result of SEUs. Figure 4 shows the fraction of data links with

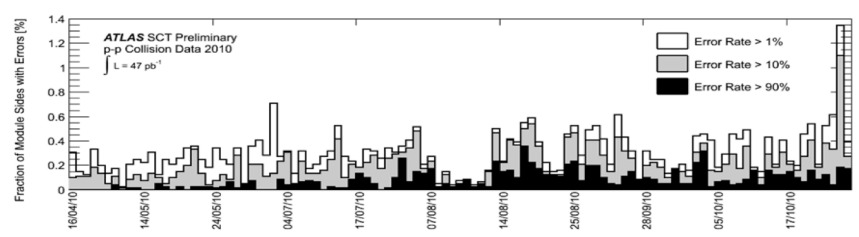

Figure 4: Data link error rate during 2010 data taking.

errors during the 2010 data taking period, which shows that the fraction of data links with errors was typically $\sim 0.2 \%$.

The main component in the SCT DAQ is the Readout Driver Board (ROD), which provides the front-end data flow, data processing and control of up to 48 modules. There are 90 RODs in the SCT DAQ, and if any experience an error condition (such as FIFO overflows), they will exert a BUSY signal to stop ATLAS data taking. In 2010, SCT implemented the mechanism to automatically reconfigure and reintegrate an entire ROD with minimal interruption to ATLAS data taking, in the very rare cases of a ROD BUSY.

\subsection{TX Issues}

A significant component failure has been the off-detector VCSEL arrays used to transmit the trigger and commands to the modules. The onset of deaths of individual VCSEL channels started several months after SCT operations began, and continued thereafter at a typical rate of three or four deaths per day. VCSEL deaths were originally attributed to inadequate ESD precautions at the point of their manufacture in the factory, and the entire operational stock of VCSELs were replaced in 2009 with units manufactured with improved ESD procedures. However, further TX channel deaths within the newly installed batches were then attributed to degradation arising from exposure of the VCSELs to humidity. In 2011, new TX transmitters from a different manufacturer demonstrated improved rebustness against humidity, and all TX transmitters were replaced again with those products after the end of 2011 operations.

Operationally, the impact of the TX deaths was minimised by the ability to use TX redundancy. If TX redundancy was not available (for example, if the module providing the redundancy was also using redundancy itself) then the module was disabled until the opportunity arose to replace the transmitter between data taking periods. The number of modules disabled due to TX deaths and the inability to use redundancy rarely increased above $\sim 5$.

\section{Tracking Performance}

\subsection{Noise}

Calibration of the SCT can be performed via charge injection in the front end of the ABCD. For each channel, a histogram of occupancy as a function of discriminator threshold is created, and a complementary error function fitted. The threshold at which the occupancy is $50 \%$ corresponds to the median of the injected charge, while the sigma gives the noise after amplification. To calibrate the discriminator threshold, the DAQ system initiates threshold scans for several different values of injected charge, and a fit to the resulting response curve yields the gain and the input noise for each channel. Typical noise

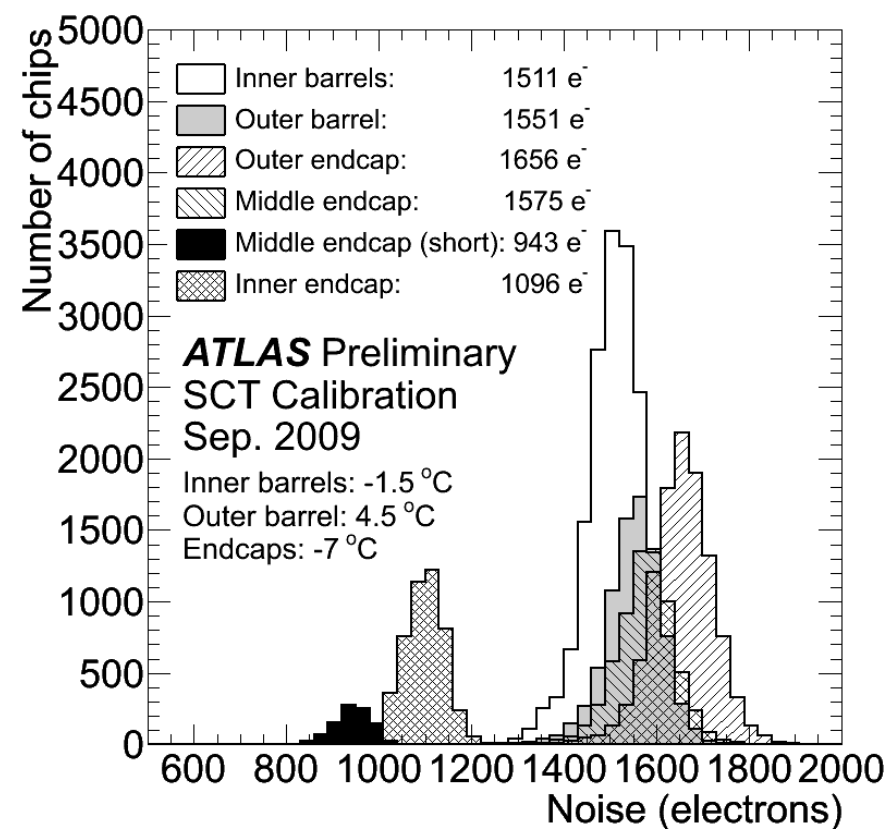

Figure 5: Typical mean chip noise for the SCT barrels and end-caps.

values are shown in Figure 5, which can be compared with a hit threshold from tracks of $\sim 6000$ electrons. The variation in noise reflects the different module shapes for the barrels and end caps. An alternative method is to determine the noise occupancy from counting the fraction of triggers which result in a hit when there is no particle activity in the detector. Figure 


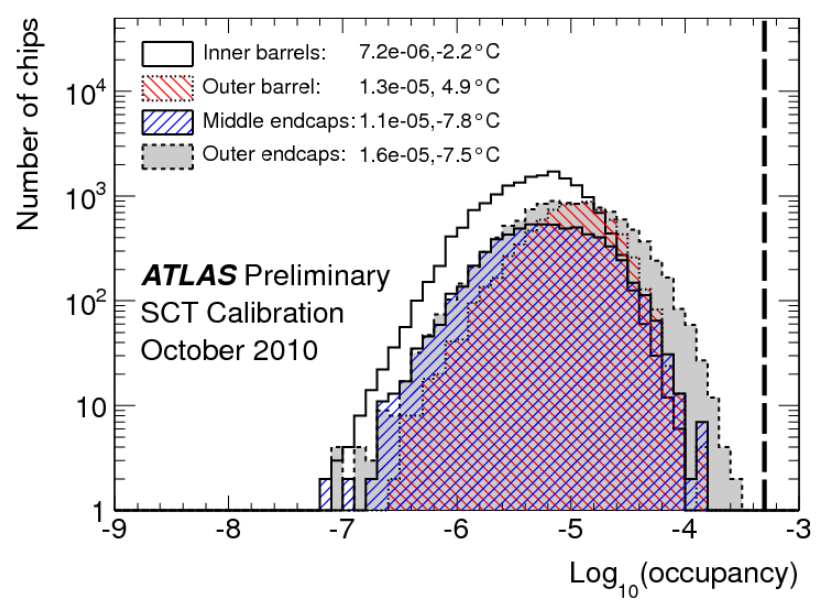

Figure 6: Noise occupancy data for the SCT barrels and end-caps. The dashed line indicates the nominal design limit.

6 shows the measured noise occupancy, which is significantly lower then the design limit of $<5 \times 10^{-4}$.

\subsection{Intrinsic Hit Efficiency}

The hit efficiency is determined by counting the number of recorded hits on high $\mathrm{P}_{T}$ tracks $(>1 \mathrm{GeV} / \mathrm{c})$ through the SCT by the number of possible hits, after ignoring known disabled strips. The nominal design requirement was for hit efficiency to be $>99 \%$. Figure 7 shows the hit efficiency for each side of the

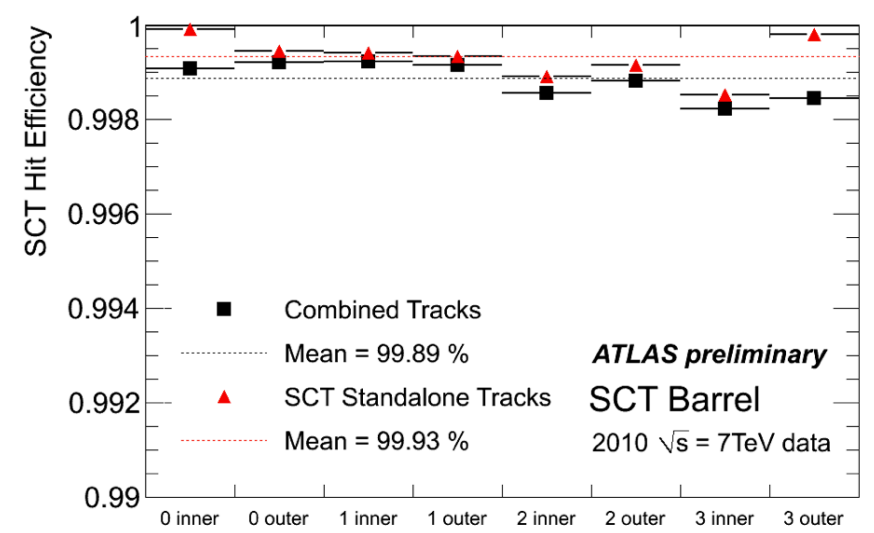

Figure 7: Hit efficiency for the 4 barrel layers, for SCT standalone tracks and tracks using information across all 3 ID systems

4 barrel layers. The barrels have have a mean hit efficiency of 99.9\% over all layers. Similarly, the hit efficiencies for the SCT end-caps are measured to be $99.8 \%$.

\subsection{Lorentz Angle}

Charge carriers drifting through the silicon bulk are deflected in the presence of a magnetic field. The number of strips which collect charge from an incident track (the "cluster width") varies with the angle of incidence of the track. The track incidence angle at which the cluster width is minimal is called the Lorentz angle, which is a function of the B-field, depletion voltage and temperature. Figure 8 shows the cluster width as a function of track incidence angle for the barrel layers, indicating the Lorentz angle. Figure 9 shows the measured Lorentz

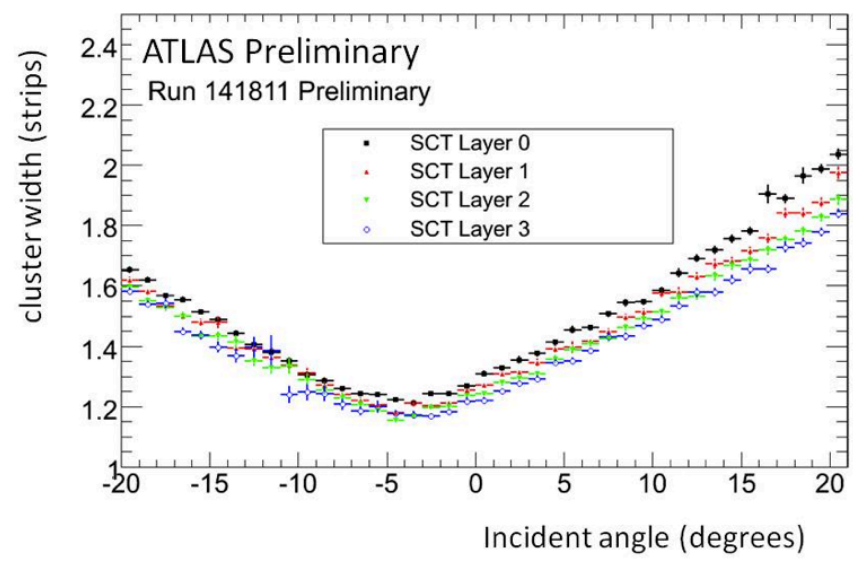

Figure 8: Cluster width size in strips vs track incident angle for the barrel layers.

angles for collision data and cosmic ray data for each barrel layer compared with model predictions, which are sensitive to the models of signal digitisation and radiation damage. Measured values are in very good agreement with predictions. Note the different value for Layer 3 is due to an increased operating temperature for this layer.

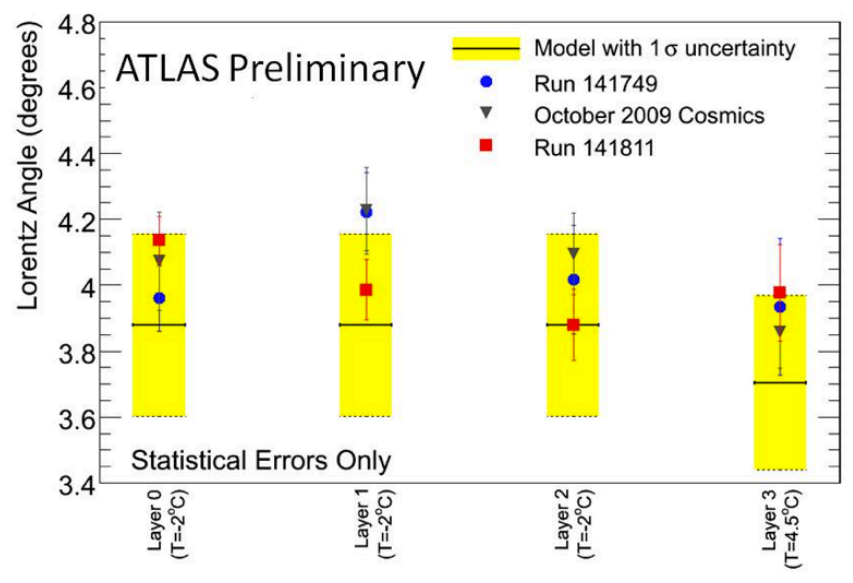

Figure 9: Measured Lorentz angle for each barrel layer from cosmic ray data and collision data, and comparison with model predictions.

\subsection{Alignment}

Alignment in SCT is performed using a track based algorithm which minimises the $\chi^{2}$ of track hit residuals (defined as the measured hit position minus the expected position from the track extrapolation). Initially, alignment was performed using survey and cosmic ray data, and continues to improve with the 
acquisition of high $\mathrm{P}_{T}$ tracks from collision data. Figure 10

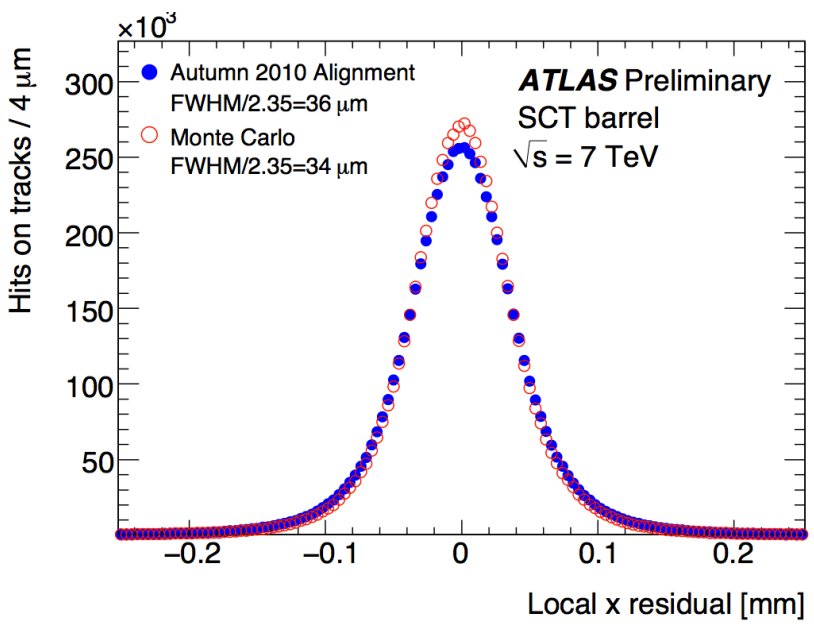

Figure 10: Unbiased residual distributions for the barrel layers, comparing measured data with Monte Carlo simulation.

shows the unbiased residual distributions in $\mathrm{x}$ for the barrel layers, comparing the ideal alignment from Monte Carlo predictions with the measured residuals from data in Autumn 2010. The FWHM of measured data was $36 \mu \mathrm{m}$ at that time and can be compared with $42 \mu \mathrm{m}$ measured in May 2010. The improvement with increased data is clear as the measured residuals approach the design value of $34 \mu \mathrm{m}$. The FWHM for end-cap data has already reached $38 \mu \mathrm{m}$ which is the ideal limit for the endcaps from Monte Carlo predictions.

\subsection{Mechanical Stability}

The SCT is equipped with a Frequency Scanning Interferometry (FSI) system which continuously monitors its mechanical stability. The FSI consists of 842 fibre coupled interferometers which form a geodetic grid of distance measurements between nodes to determine the relative positions and orientations of the barrel cylinders and end-cap disks. The FSI has demonstrated that the SCT is mechanically stable and rigid. In the absence of any environmental changes, the interferometer lengths are very stable at $\sigma \sim 9 \mathrm{~nm}$. Typical deviations during a ramp of the solenoid magnet (where the $2 \mathrm{~T}$ solenoidal field cycles off then on again) result in temporary deviations of up to $\sim 3 \mu \mathrm{m}$.

\section{Radiation Damage}

Significant increases in leakage currents have been observed in the SCT, as expected from bulk damage due to non-ionising radiation. Figure 11 shows the average leakage current for modules from each of the barrel layers measured over a two year period. The leakage current increase correlates closely with the delivered LHC luminosity and temperature cycles, and there is excellent agreement with leakage current predictions derived from the temperature profiles and fluence from FLUKA[7] simulations.

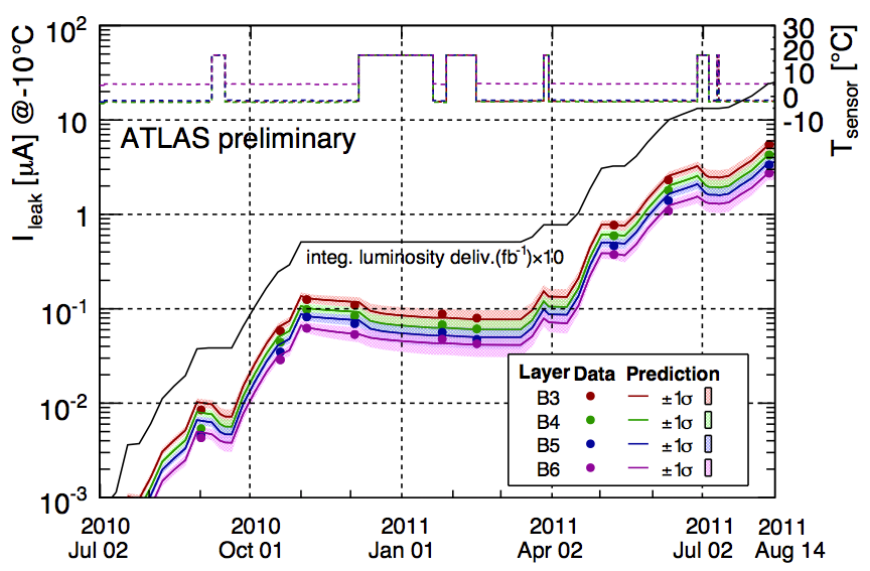

Figure 11: SCT barrel leakage currents during 2010 and 2011, showing correlations with delivered luminosity and temperature, and compared to predictions from Monte Carlo.

\section{Summary}

The SCT has been fully operational throughout all ATLAS data taking operations. The detector acceptance and operational efficiency are both excellent, and all tracking performance indicators have met or significantly exceed design specifications. The affects of radiation damage so far match closely the predictions based on the delivered luminosity and temperature history.

\section{References}

[1] J. Carter et al, The silicon microstrip sensors of the ATLAS semiconductor tracker, Nucl. Instrum. Meth. A578 98-118 (2007).

[2] J. Carter et al, The barrel modules of the ATLAS semiconductor tracker, Nucl.Instrum.Meth. A568, 642-671 (2006).

[3] P.Allport et al, The ATLAS semiconductor tracker end-cap module, Nucl.Instrum.Meth. A575, 353-389 (2007)

[4] F. Campabadal et al, Design and performance of the ABCD3TA ASIC for readout silicon strip in the ATLAS semiconductor tracker, Nucl.Instrum.Meth. A552, 292-328 (2005).

[5] T.Weidberg et al, The optical links of the ATLAS SemiConductor Tracker, JINST 2, P09003 (2007).

[6] A. Barr et al, The data acquisition and calibration system for the ATLAS Semiconductor Tracker, JINST 3 P01003 (2008).

[7] A. Ferrari, P. Sala, A. Fasso and J. Ranft, FLUKA: A multi-particle transport code, CERN-2005-10 (2005), INFN/TC-05/11, SLAC-R-773. 